\title{
Mobile phone use, exposure to radiofrequency electromagnetic field, and brain tumour: a case-control study
}

\author{
T Takebayashi', N Varsier ${ }^{2,3}$, Y Kikuchi', K Wake ${ }^{3}$, M Taki $^{2}$, S Watanabe ${ }^{3}$, S Akiba ${ }^{4}$ and N Yamaguchi",5 \\ 'Department of Preventive Medicine and Public Health, Keio University School of Medicine, Tokyo, Japan; ${ }^{2}$ Department of Electrical and Electronic \\ Engineering, Tokyo Metropolitan University, Tokyo, Japan; ${ }^{3} E M C$ Group, Applied Electromagnetic Engineering, National Institute of Information and \\ Communications Technology, Tokyo, Japan; ${ }^{4}$ Department of Epidemiology and Preventive Medicine, Kagoshima University Graduate School of Medical \\ and Dental Sciences, Kagoshima, Kagoshima; ${ }^{5}$ Department of Public Health, Tokyo Women's Medical University, Tokyo I 62-8666, Japan
}

In a case-control study in Japan of brain tumours in relation to mobile phone use, we used a novel approach for estimating the specific absorption rate (SAR) inside the tumour, taking account of spatial relationships between tumour localisation and intracranial radiofrequency distribution. Personal interviews were carried out with 88 patients with glioma, I 32 with meningioma, and I02 with pituitary adenoma (322 cases in total), and with 683 individually matched controls. All maximal SAR values were below 0.1 W kg ${ }^{-1}$, far lower than the level at which thermal effects may occur, the adjusted odds ratios (ORs) for regular mobile phone users being 1.22 ( $95 \%$ confidence interval (Cl): $0.63-2.37)$ for glioma and $0.70(0.42-1.16)$ for meningioma. When the maximal SAR value inside the tumour tissue was accounted for in the exposure indices, the overall OR was again not increased and there was no significant trend towards an increasing OR in relation to SAR-derived exposure indices. A non-significant increase in OR among glioma patients in the heavily exposed group may reflect recall bias.

British Journal of Cancer (2008) 98, 652-659. doi:10.1038/sj.bjc.66042I4 www.bjcancer.com

Published online 5 February 2008

(c) 2008 Cancer Research UK

Keywords: glioma; meningioma; mobile phone; case-control study; epidemiology

The rapid increase in mobile phone use has raised public concern about their safety (Rothman et al, 1996; Violanti and Marshall, 1996; Repacholi, 1998; Blettner and Berg, 2000; Rothman, 2000). Since only glial and meningial tissue close to the surface of the head is exposed to relatively high electromagnetic fields (EMFs) emitted from mobile phones, brain tumours, especially glioma and meningioma, have received particular attention, along with acoustic neurinoma and salivary gland tumours. To investigate whether mobile phone users have an increased risk for these tumours, a collaborative case-control study in 13 countries, the INTERPHONE study, was initiated in 2000, coordinated by the International Agency for Research on Cancer (IARC) and is still underway (Cardis and Kilkenny, 1999; Christensen et al, 2005; Lönn et al, 2005; Hepworth et al, 2006; Schuz et al, 2006; Takebayashi et al, 2006; Cardis et al, 2007; Hours et al, 2007). Some national reports are already published, with mixed findings.

A central issue has been how precisely to estimate the actual EMF exposure, given the necessary reliance on self-reported use. Different parts of the brain are known to be exposed to EMFs of different magnitudes, related not only to which ear the phone is placed on, but also to the characteristics of different mobile phone models. The specific absorption rate (SAR) is widely accepted as a dosimetric quantity in guidelines on EMF exposure (e.g., the

*Correspondence: Dr N Yamaguchi;

E-mail: yamaguch@research.twmu.ac.jp

Received 17 September 2007; revised 19 December 2007; accepted 4 January 2008; published online 5 February 2008
International Commission on Non-Ionizing Radiation Protection (ICNIRP), 1998) in frequency ranges including those used for mobile phones. The SAR, representing absorbed radiofrequency (RF) power per unit mass of body tissue, is closely related to thermal effects. If non-thermal effects are involved, SAR is also relevant, being as it is closely correlated with internal electric and magnetic fields in tissue near the radiation source (Mochizuki et al, 2002).

We have investigated whether mobile phone use increased brain tumour risk in Japan. The study followed the common, core protocol of the INTERPHONE study, but for estimating RF exposure level in different areas of the brain in light of its great variability, we adopted a new approach using a heterogeneous head model (Japanese numerical TARO model's head) (Nagaoka et al, 2004).

\section{METHODS}

The study area encompassed Tokyo, consisting of 23 wards (the metropolitan area) and 14 cities (the municipal area), along with 25 adjacent cities. In a preliminary survey, it was found that 30 of the 172 hospital neurosurgery departments in Tokyo treated approximately $90 \%$ of brain tumours in the area. Of these 30 departments, 21 agreed to participate in the study, and so it was estimated that about $75 \%$ of the meningioma and glioma cases in the study area would be covered. There were no differences in patients' socioeconomic status between participating and nonparticipating hospitals. The case group consisted of newly diagnosed meningiomas, gliomas, and pituitary adenomas at ages 
30-69 years treated in participating hospitals, with case recruitment performed prospectively from December 2000 to November 2004. Participation was $58.7 \%$ (88 out of 150 ) for glioma, $77.6 \%$ (132 out of 170) for meningioma, and 75.6\% (102 out of 135) for pituitary adenoma. The main reason for non-participation was failure to contact the patients; for example, only $5 \%$ of glioma eligible patients refused, whereas the rest (37\%) were not informed of the study during their hospital stay by their attending physicians. The 10 cases found to have been diagnosed before June 2000, more than 6 months before the study began, were excluded from the analysis (with their controls) leaving 83 gliomas, 128 meningiomas, and 101 pituitary adenomas. Cases treated surgically were verified histopathologically (codes of ICD-O 3rd ed.: glioma, 9380-9384, 9390-9394, 9400-9401, 9410-9411, $9420-9424,9430,9440-9443,9450-9451,9460,9480-9481$; meningioma, 9530-9539), but some were diagnosed by magnetic resonance imaging. Among the 83 gliomas and 128 meningiomas, 78 (94\%) and 118 (92.2\%), respectively, underwent surgery. The date of diagnosis was defined as when the tumour was first identified radiologically.

Controls were selected from the general population by random digit dialling, in which phone numbers for home fixed-phones were generated randomly. Volunteers to act as controls were sought until at least one for each case was identified who agreed to participate; they were individually matched for age (within a 5-year range), sex, and residence with the cases. Participation among contacted controls was $52.5 \%$ (196 out of 373 ) for glioma, $51.6 \%$ (279 out of 541) for meningioma, and $49.4 \%$ (208 out of 421) for pituitary adenoma. A brief questionnaire was administered over the phone or via a self-administered paper questionnaire to those who did not agree to face-to-face interviews, to compare phone use between participants and non-participants among eligible controls. Basic information on age, sex, brief history of mobile phone use, and some life-style factors was available for $75.6,73.0$, and $72.0 \%$ of the control for glioma, meningioma, and pituitary cases, respectively.

Each case subject and matched control was interviewed by the same nurse or other health professional specifically trained for this study. A Japanese version of the computer-assisted interview system developed for the INTERPHONE study was used for the face-to-face interviews (Cardis and Kilkenny, 1999). Subjects were asked about their mobile phone use, including the dates of starting and stopping to use each phone, the average duration and frequency of calls, and other usage patterns in chronological order. Use of the Personal Handy-phone System (PHS), a type of cordless telephone system, was also recorded. Demographic variables, medical history, and occupational history were also recorded. Clinical information on the cases was obtained from the relevant department.

When a case had over four matched controls, the latter was reduced randomly to make a 4:1 ratio for each case. Thus, 83 gliomas had 163 matched controls, 128 meningiomas had 229 controls, and 101 pituitary adenomas had 161 controls.

Regular mobile phone use was defined as used at least once a week for 6 months, and the reference date for phone use was set at one year before diagnosis for each case to eliminate any effect of disease in its prodromal stage; the same date being applied to matched controls. Furthermore, for regular phone users, two indices were created: cumulative length of use and cumulative call time, the former defined as the time (years) since first use, excluding any period when it was not regularly used. For recent use, either the reference date or the stop date of the last phone, whichever came first, was adopted as the end of use date. The cumulative call time was the total call durations (hours) since first use, which was the sum of call durations with all phones. Typically, the daily call duration was calculated by multiplying the average call duration per call by the number of calls per day, the call duration for each phone being the product of the daily call duration and the length of use.
An odds ratio (OR), in which the reference category was the non-user, unless otherwise specified, was calculated with the conditional logistic regression model, in which the educational level (junior high school, high school, 2-year college, 4-year college or graduate school) and marital status (married or others) were simultaneously adjusted for as categorical variables.

To examine for an association with laterality of phone use, we employed a conditional logistic regression analysis, in which EMF exposure was assumed only when the self-reported side of phone use was the same as the tumour's (ipsilateral use). A similar analysis was performed for contralateral use, with exposure assumed when the self-reported side of phone use was the opposite of the tumour's. If both ears were reported used for phone use, exposure was assumed for both analyses.

To account for the three-dimensional, spatial relationship between tumour and RF exposure distribution, we attempted to estimate the maximal SAR value inside the tumour. Since pituitary adenomas occur in the sellar region, where RF exposure is negligible, SAR was estimated only for gliomas and meningiomas as follows. Mobile phones were categorised into a small number of groups in terms of SAR distribution (Wake et al, 2006), since each phone model has a different intracranial SAR distribution and it is impossible to estimate the actual SAR distributions for the hundreds of mobile phones used by the cases and controls. We used the SAR distribution data from 76 phones on the Japanese market in January 2001 measured with a phantom using standard procedure for compliance testing in Japan similar to the international standard procedure (IEC 62209). Surface SAR was measured in a limited region near the phone, and cube SAR data was measured in a small three-dimensional region around the maximal SAR location. The cluster analysis was then applied to categorise the phones, in which SAR distributions were represented by location of maximal SAR, surface area larger than $50 \%$ of the maximal SAR, or depth larger than $50 \%$ of the maximal SAR. The results of categorisation depended on the conditions of phone use. Mobile phones could be classified into four categories assuming normal use condition as cheek position with antenna extracted: (1) flip/flop phones of both $800-\mathrm{MHz}$ and $1.5-\mathrm{GHz}$ band with an antenna in the centre; (2) flip/flop phones of $800-\mathrm{MHz}$ band with an antenna on the top; (3) $1.5-\mathrm{GHz}$ band phones with an antenna on top; and (4) straight phones of $800-\mathrm{MHz}$ band with an antenna on top. On the basis of this finding, a hypothetical threedimensional SAR distribution was constructed for each category of mobile phone on the above head model, Japanese numerical TARO model's head (Nagaoka et al, 2004). First, we estimated threedimensional SAR distribution for each phone in TARO model's head with the measured SAR data (Wake et al, 2005). The surface SAR data measured in the limited regions of the phantom was extrapolated to its whole head, and was further projected to the surface of TARO model's head. Then, SAR in depth direction was estimated assuming exponential decay of the first three layers. Finally, SAR distribution for each phone category was obtained by averaging SAR distributions for all phones belonging to the category (Varsier et al, 2007).

Each mobile phone actually used was allocated to one of four categories and the hypothetical SAR distribution assigned, taking into account which ear was in contact (Varsier et al, 2007). The tumour location for each case was measured on a 12 computed tomography scan-cut chart model. Because of their differences, the TARO's head was transformed to match the chart model by projection. Then the SAR value inside the tumour was estimated at a $1-\mathrm{cm}$ resolution and the maximal SAR was identified for each phone. For matched controls, the maximal SAR value was estimated for the tumour location of the corresponding cases.

Three exposure indices were constructed based on the SAR distribution inside the tumour. The mean maximal SAR (mean maxSAR) was calculated for each subject by averaging the maximal SAR value over the mobile phone used by the subject. The 
cumulative maxSAR-year was defined as the cumulative years of use weighted by maxSAR, or calculated by summing the product of maxSAR and length of use in years for each phone over the different mobile phones used by each subject. The cumulative maxSAR-hour was defined as the cumulative call time weighted by maxSAR, or calculated by summing the product of the maxSAR and call time in hours for each phone over the different mobile phones used by each subject.

In a case-only analysis, the mean maxSAR, cumulative maxSARyear, and cumulative maxSAR-hour were estimated for the actual and for a hypothetical tumour location, which was the mirror image of the tumour on the opposite side across the sagittal plane. The rationale was that the above SAR indices should have a higher value for the actual than for the hypothetical tumour location if RF exposure increased tumour risk. Each of the three indices was dichotomised into high and low values using the median distribution among the controls as the cut-off point: 0.0012 (glioma) and 0.0011 (meningioma) for average maxSAR; 0.0059 (glioma) and 0.0041 (meningioma) for cumulative maxSAR-year; and 0.447 (glioma) and 0.146 (meningioma) for cumulative maxSAR-hour; the McNemar test was used for comparisons.

Statistical analyses used STATA/SE version 8.2 (College Station, StataCorp, TX, USA). All statistical tests were two-sided; separate analyses were performed including and excluding the PHS system, which has a much lower emission power than mobile telephones, and as the results showed no substantial differences (data not shown), those analyses excluding the PHS system are presented here.

This study was approved by the Institutional Review Boards of the participating institutes. Its protocol was reviewed by the research group's Privacy Protection Subcommittee, which also monitored the procedures employed.

\section{RESULTS}

The basic characteristics of the cases and controls are shown in Table 1. For time-varying items, the situations at the reference date are displayed. Cases and controls showed no substantial differences with respect to age, sex, residential area, educational level, or marital status. Educational level and marital status were considered to reflect their socioeconomic status and were selected $a$ priori as confounding variables. The overall participation rate of the controls in the full study was $51.2 \%$, but an additional $28.8 \%$ of eligible controls answered the brief survey about their mobile phone use. The age- and sex-adjusted proportions of regular users were $66.4 \%$ (glioma) and $55.1 \%$ (meningioma) in the brief survey, and were comparable with $65 \%$ (glioma) and 51.5\% (meningioma) in the full study.

The adjusted OR (95\% confidence interval (CI)) for regular mobile phone use was $1.22(0.63-2.37)$ for glioma, $0.70(0.42-$ 1.16) for meningioma, and $0.90(0.50-1.61)$ for pituitary adenoma,

Table I Case-control comparison of basic characteristics at reference date

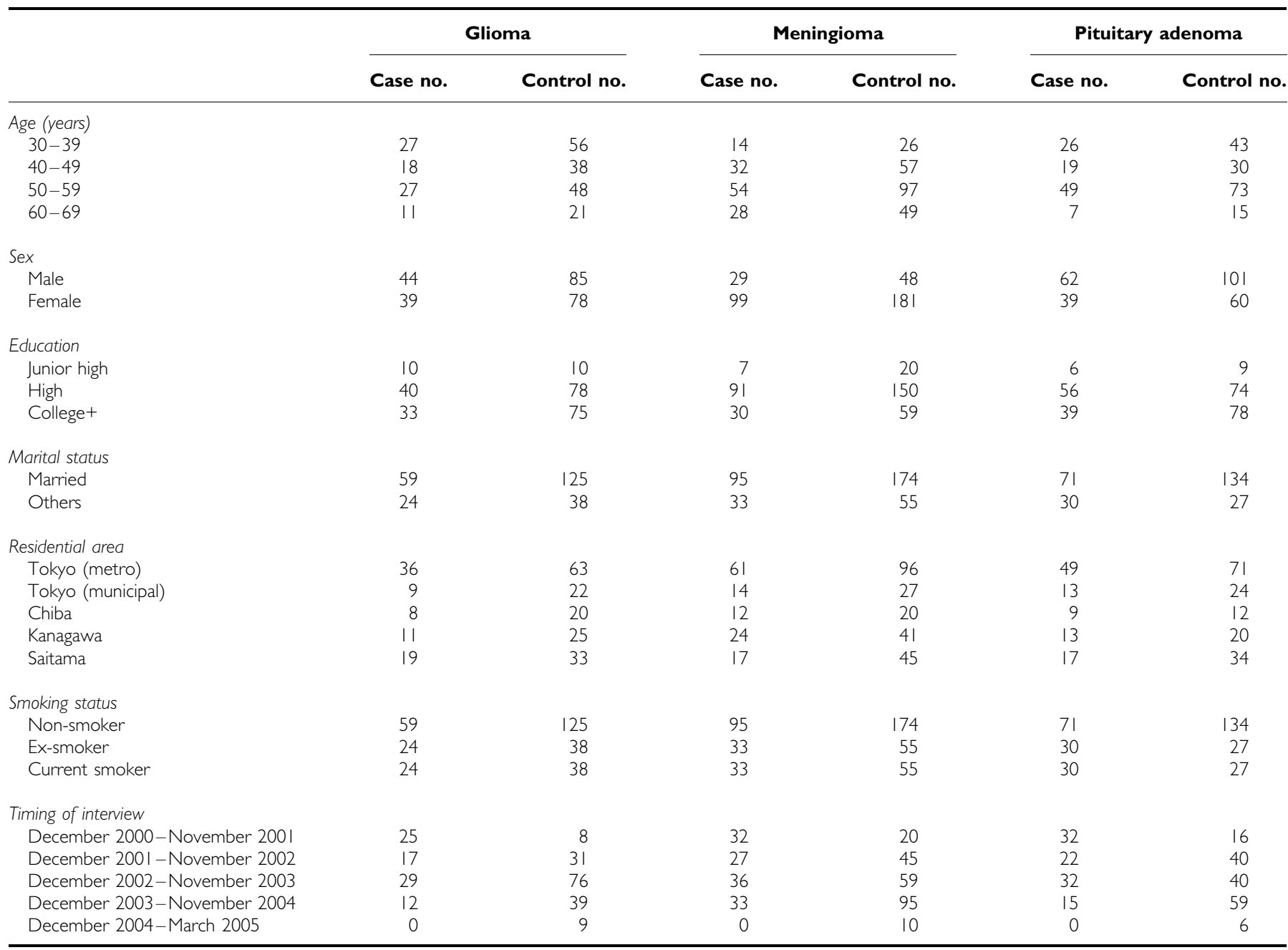


Table 2 Case-control comparison of the indices of mobile phone use

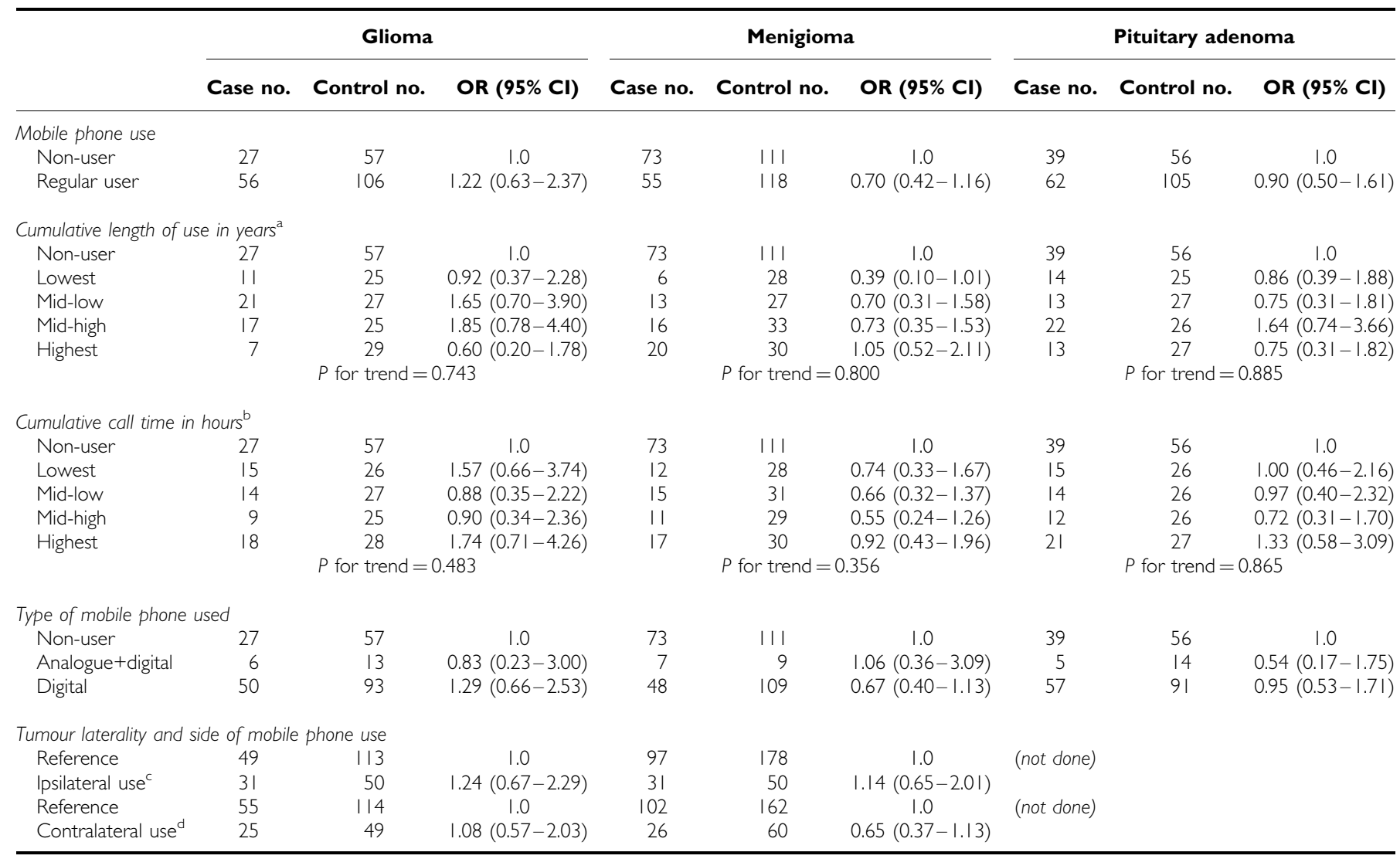

Abbreviations: $\mathrm{Cl}=$ confidence interval; $\mathrm{OR}=$ odds ratio adjusted for education and marital status. ${ }^{\mathrm{a}} \mathrm{Cut}$-off points for quartiles (divided on the basis of the distribution of the

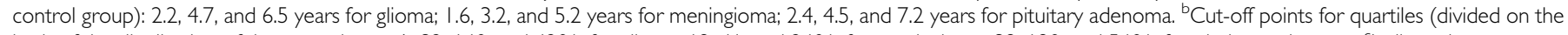
basis of the distribution of the control group): 32, 160, and $620 \mathrm{~h}$ for glioma; 19, 61, and $260 \mathrm{~h}$ for meningioma; 39, 190, and $560 \mathrm{~h}$ for pituitary adenoma. Ipsilateral use: tumour location (left/right) was the same as the side of mobile phone use. ${ }^{\mathrm{d} C o n t r a l a t e r a l ~ u s e: ~ t u m o u r ~ l o c a t i o n ~(l e f t / r i g h t) ~ w a s ~ t h e ~ o p p o s i t e ~ a s ~ t h e ~ s i d e ~ o f ~ m o b i l e ~ p h o n e ~ u s e . ~}$

the reference date being set at 1 year before diagnosis (Table 2). The adjusted OR did not change significantly when the reference date was set at 5 years before diagnosis: $0.90(0.47-1.72)$ for glioma, $1.32(0.72-2.43)$ for meningioma, and $0.96(0.52-1.79)$ for pituitary adenoma.

When cumulative length of use and call time were categorised into quartiles according to distribution among the controls for each tumour type, as shown in Table 2, no increasing trend was found in risks of glioma, meningioma, or pituitary adenoma. In addition, the OR for a cumulative use of 10 years or longer was $0.58(0.09-3.86)$ for glioma, $1.35(0.31-5.93)$ for meningioma, and $1.15(0.22-5.18)$ for pituitary adenoma, although the numbers were very small: 2 cases and 6 controls for glioma; 4 cases and 4 controls for meningioma; and 4 cases and 5 controls for pituitary adenoma. The OR for a cumulative call time of $2000 \mathrm{~h}$ or more was $1.47(0.41-5.28)$ for glioma, $0.64(0.14-3.86)$ for meningioma, and $1.41(0.46-4.37)$ for pituitary adenoma, again on the basis of very small numbers: 6 cases and 9 controls for glioma; 3 cases and 6 controls for meningioma; and 9 cases and 12 controls for pituitary adenoma.

No subject had used analogue-type phones alone. No difference in OR was identified for use of both analogue and digital phones $v s$ digital phones only: $0.83(0.23-3.00)$ vs $1.29(0.66-2.53)$ for glioma; $1.06(0.36-3.09)$ vs $0.67(0.40-1.13)$ for meningioma; and $0.54(0.17-1.75) \quad v s \quad 0.95(0.53-1.71)$ for pituitary adenoma, respectively.

In the laterality analysis, in which it was simply assumed that exposure existed when the tumour location (left or right) matched that for phone use, the ORs for ipsilateral and contralateral use were $1.24(0.67-2.29)$ and $1.08(0.57-2.03)$ for glioma, and 1.14 $(0.65-2.01)$ and $0.65(0.37-1.13)$ for meningioma, respectively.

For the analysis using maxSAR-derived exposure indices, 77 of 83 glioma cases and 125 of 128 meningioma cases with tumour location charts available, with 151 and 221 controls, respectively, were included. Mean maxSAR was estimated for each case, ranging from $1.2 \times 10^{-6}$ to $0.0599 \mathrm{~W} \mathrm{~kg}^{-1}$ for glioma and $6.8 \times 10^{-7}$ to $0.0619 \mathrm{~W} \mathrm{~kg}^{-1}$ for meningioma (Figure 1). The distribution of cumulative maxSAR-year and cumulative maxSAR-hour are also presented in Figure 1. The non-exposed group consisted of nonusers and regular users whose mean maxSAR was extremely low (less than $\left.1 \times 10^{-4}\right)$ : $34(44.2 \%)$ cases and $71(47.0 \%)$ controls for glioma, and $81(64.8 \%)$ cases and $127(75.5 \%)$ controls for meningioma. The OR for exposed to non-exposed was 1.28 (0.63$2.57)$ for glioma and $0.72(0.42-1.24)$ for meningioma. In addition, the exposed group was divided into four subgroups on the basis of the quartiles of distribution of maxSAR-derived indices among the controls. No increasing trend was found for either glioma or meningioma risk with an increase in mean maxSAR, cumulative maxSAR-year, or cumulative maxSAR-hour (Table 3 ).

Since the ORs for the highest quartile were all higher than unity for glioma (1.04 (0.37-2.93) for mean maxSAR, $1.75(0.63-4.85)$ for cumulative maxSAR-year, and $1.55(0.57-4.19)$ for cumulative maxSAR-hour), we further examined risk associated with a very high exposure. When categorised by cut-off points of 0.001 and $0.01 \mathrm{~W} \mathrm{~kg}^{-1}$, the adjusted OR for those with mean maxSAR over $0.01 \mathrm{~W} \mathrm{~kg}^{-1}$ as compared with the non-exposed was $0.87(0.28-$ $2.75)$ for glioma and $1.17(0.40-3.39)$ for meningioma, indicating no substantial increase in risk. When categorised by cut-off points 
A Glioma

a

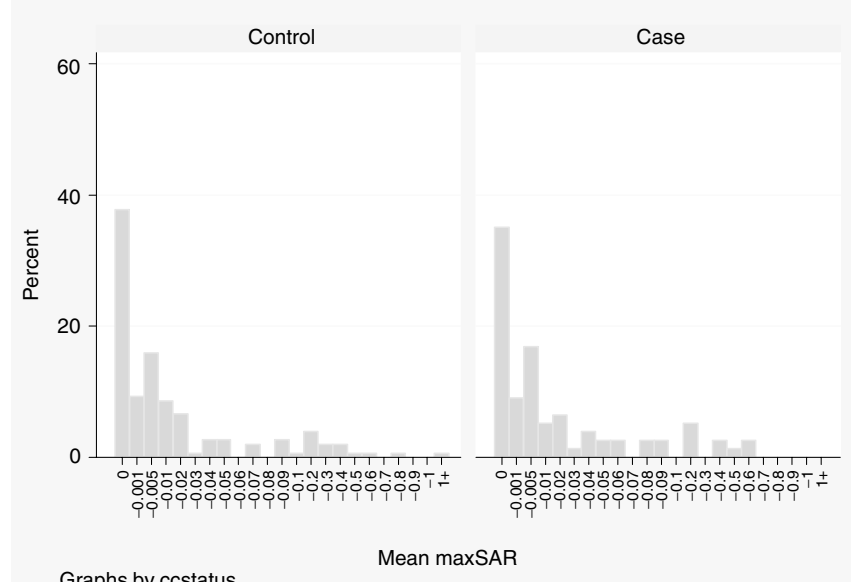

b

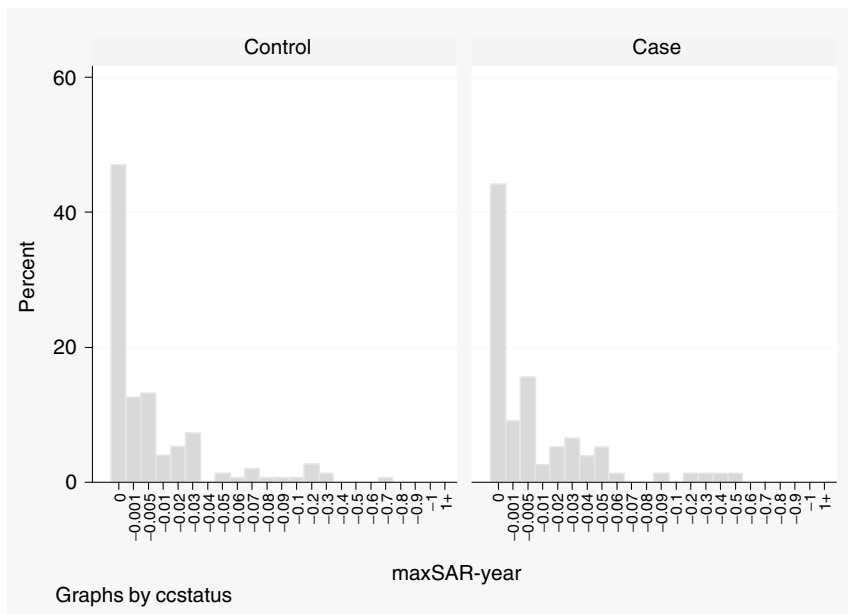

C

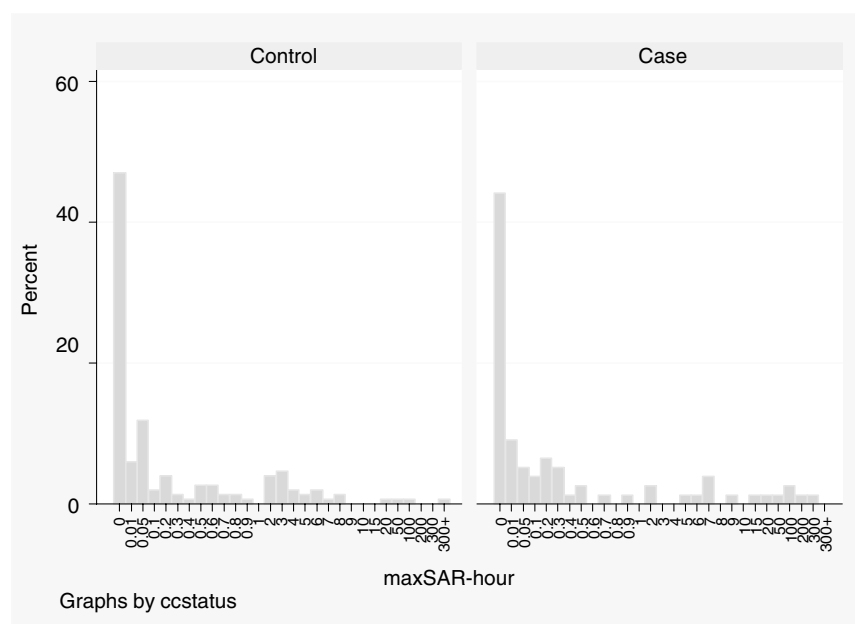

B

a Meningioma

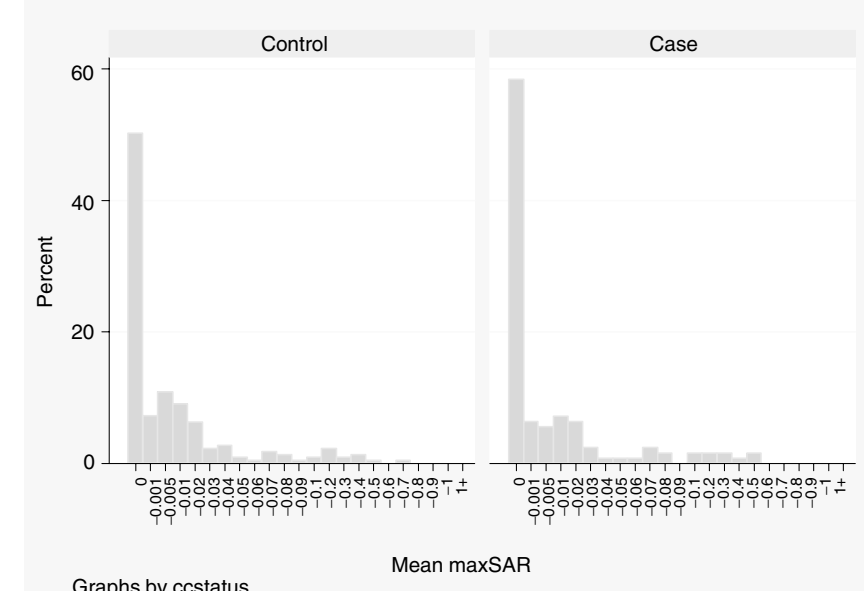

b


c

Cumulative maxSAR-hour

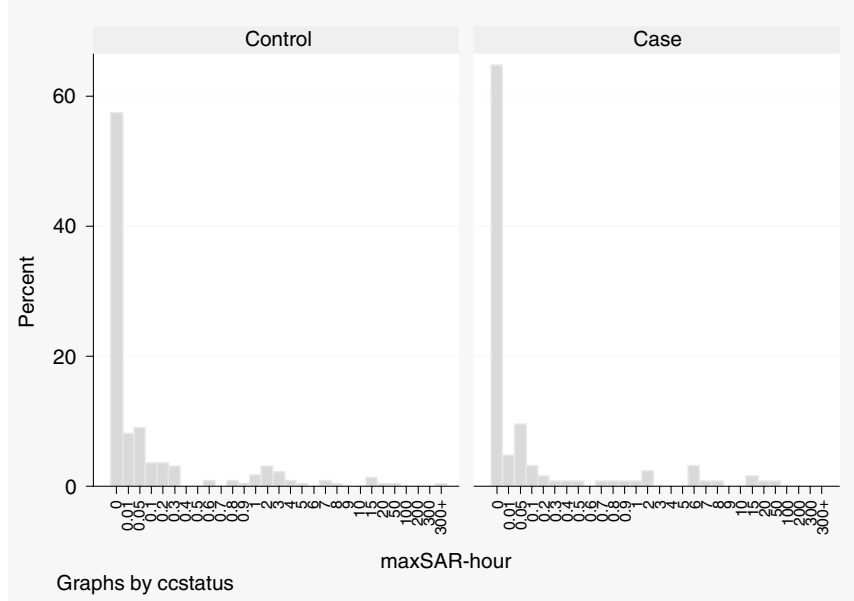

Figure I Distribution of estimated maxSAR-derived exposure indices in the tumour. (A) Glioma; (a) mean maxSAR; (b) cumulative maxSAR-year; (c) cumulative maxSAR-hour. (B) Meningioma; (a) mean maxSAR; (b) cumulative maxSAR-year; (c) cumulative maxSAR-hour.

of $0.001,0.01$, and $0.1 \mathrm{~W} \mathrm{~kg}^{-1}$-year, the adjusted OR for those with cumulative maxSAR-year over $0.1 \mathrm{~W} \mathrm{~kg}^{-1}$-year as compared with the non-exposed was $0.63(0.14-2.93)$ for glioma and $2.72(0.47-$
15.98) for meningioma, again indicating no substantial increase in risk. When categorised by cut-off points of $0.1,1$, and $10 \mathrm{~W} \mathrm{~kg}^{-1}$ hour, on the other hand, the adjusted OR for the highest $v s$ lowest 
Table 3 Risk of brain tumour with relation to mobile phone use, considering estimated maximal SAR in the tumour as an exposure index

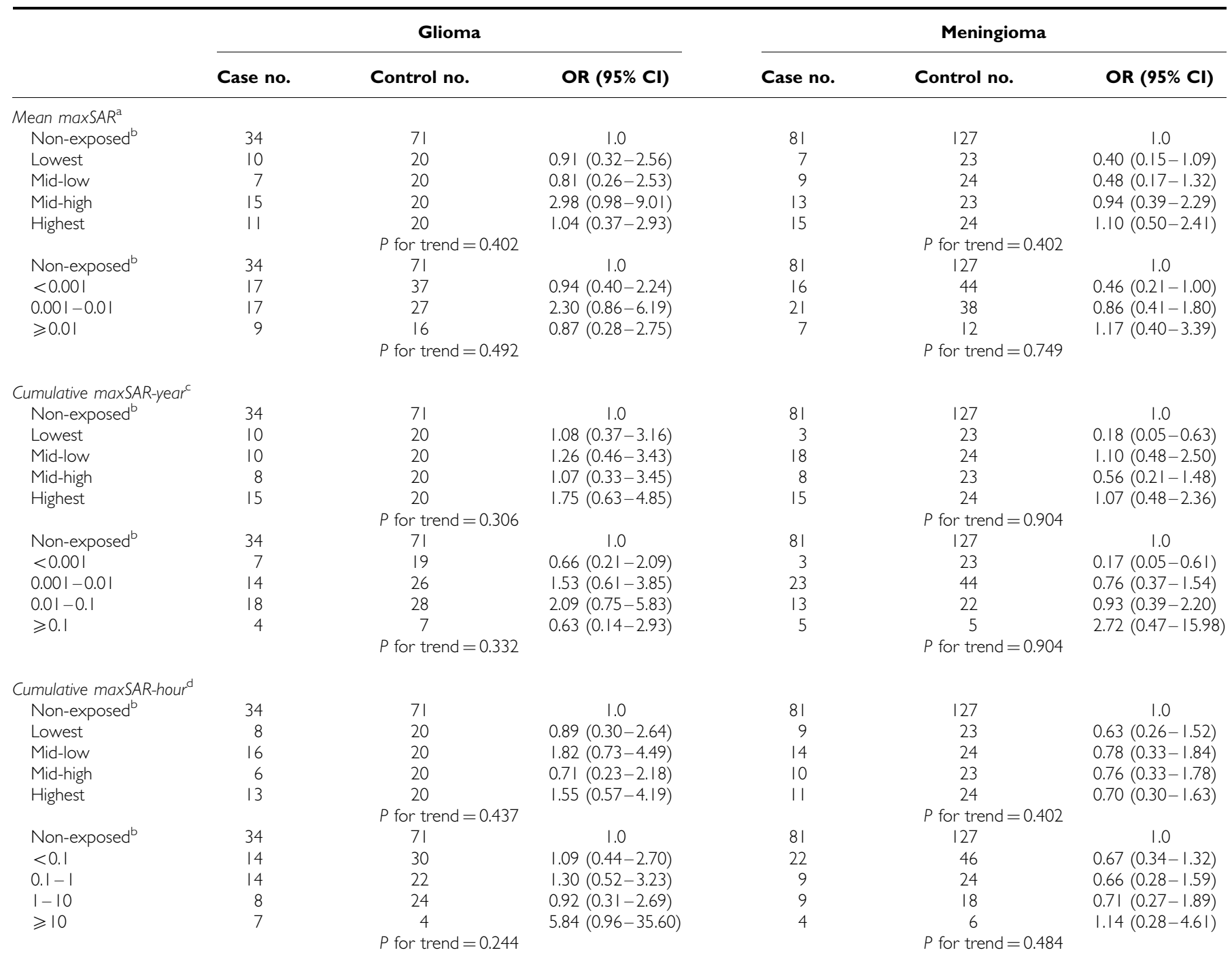

Abbreviations: $\mathrm{Cl}=$ confidence interval; $\mathrm{OR}=$ odds ratio adjusted for education and marital status; $\mathrm{SAR}=$ specific absorption rate. ${ }^{\mathrm{a} C u t-o f f s}$ for quartiles (on the basis of the distribution of the control group): $0.00036,0.0012$, and 0.008 for glioma; $0.00049,0.001 \mathrm{I}$, and 0.0048 for meningioma. ${ }^{b}$ Non-exposed group includes mobile phone users whose maximal SAR was estimated to be $<0.000 \mathrm{I} \mathrm{W} \mathrm{kg}{ }^{-1}$. ' Cut-offs for quartiles (on the basis of the distribution of the control group): $0.0012,0.0059$, and 0.025 for glioma; 0.00 I, $0.004 \mathrm{I}$, and 0.014 for meningioma. ${ }^{d}$ Cut-offs for quartiles (on the basis of the distribution of the control group): $0.028,0.447$, and 2.18 for glioma; $0.014,0.146$, and I.I2 for meningioma.

category increased, although not significantly, to $5.84(0.96-35.60)$ for glioma, on the basis of 7 cases $(9.1 \%)$ and 4 controls $(2.7 \%)$ in the highest category. No increase in risk was found for meningioma: $1.14(0.28-4.61)$, on the basis of 4 cases $(3.2 \%)$ and 6 controls $(2.7 \%)$.

In the case-only analysis, no differences were identified when three dichotomised maxSAR-derived indices were compared between the actual and hypothetical opposite tumour location (as shown in Table 4).

\section{DISCUSSION}

No consistent increase was observed in the overall risk of glioma or meningioma among mobile phone users, nor increasing trend in risk in relation to cumulative length of use or cumulative call time. We further estimated the SAR in the intracranial space with a resolution of $1 \mathrm{~cm}^{3}$, and constructed three SAR-derived exposure indices (mean maxSAR, cumulative maxSAR-year, and cumulative maxSAR-hour) to estimate the cumulative exposure level inside the tumour as precisely as possible. Again, no substantial increase in risk was observed for glioma or meningioma. Maximal SAR inside the tumour was estimated to be lower than $0.1 \mathrm{~W} \mathrm{~kg}^{-1}$ in all eligible cases, far below the ICNIRP's recommended value of $2 \mathrm{~W} \mathrm{~kg}^{-1}$ for localised SAR (head and trunk) in the general population.

This is to our knowledge, the first epidemiological study to take into account the different exposure levels inside the intracranial space. Exposure is localised close to the relevant ear, whereas glioma and meningioma develop at a variety of sites. Estimation of the actual exposure level inside the tumour is essential, therefore, to avoid exposure misclassification, which bias risk estimates towards the null. Nevertheless, recall bias is a possibility and awareness of tumour location among the cases might have affected their recall concerning which ear they used for mobile phone calls. The non-significant increase in OR of 5.84 for those with cumulative maxSAR-hour over $10 \mathrm{~W} \mathrm{~kg}^{-1}$-hour as compared with the non-exposed group could reflect recall bias, since increased OR was observed only for this heavily exposed group; it needs to be interpreted with caution. 
Table 4 Case-only analysis to compare the distribution of maximal SAR-related indices within the tumour and its axis-symmetrical location ${ }^{\text {a }}$

\begin{tabular}{|c|c|c|c|c|c|}
\hline & \multicolumn{2}{|c|}{ Glioma } & & \multicolumn{2}{|c|}{ Meningioma } \\
\hline & \multicolumn{2}{|c|}{ Axis-symmetrical tumour location ${ }^{b}$} & & \multicolumn{2}{|c|}{ Axis-symmetrical tumour location ${ }^{b}$} \\
\hline \multicolumn{6}{|l|}{ Mean maxSAR } \\
\hline & $\geqslant 0.0012$ & $<0.0012$ & & $\geqslant 0.0011$ & $<0.0011$ \\
\hline Actual tumour $\geqslant 0.0012$ & | | & 15 & Actual tumour $\geqslant 0.0011$ & 17 & II \\
\hline Location $<0.0012$ & $\mid 1$ & $\begin{array}{c}13 \\
P=0.433\end{array}$ & Location $<0.0011$ & 10 & $\begin{array}{c}14 \\
P=0.827\end{array}$ \\
\hline \multicolumn{6}{|l|}{ Cumulative maxSAR-year } \\
\hline & $\geqslant 0.0059$ & $<0.0059$ & & $\geqslant 0.0041$ & $<0.0041$ \\
\hline Actual tumour $\geqslant 0.0059$ & | | & 12 & Actual tumour $\geqslant 0.0041$ & 12 & | | \\
\hline Location < 0.0059 & 10 & $\begin{array}{c}17 \\
P=0.670\end{array}$ & Location <0.004l & 12 & $\begin{array}{c}17 \\
P=0.835\end{array}$ \\
\hline \multicolumn{6}{|l|}{ Cumulative maxSAR-hour } \\
\hline & $\geqslant 0.447$ & $<0.447$ & & $\geqslant 0.146$ & $<0.146$ \\
\hline Actual tumour $\geqslant 0.447$ & 10 & 9 & Actual tumour $\geqslant 0.146$ & 9 & 12 \\
\hline Location $<0.447$ & 9 & $\begin{array}{c}22 \\
P=1.000\end{array}$ & Location $<0.146$ & 9 & $\begin{array}{c}22 \\
P=0.664\end{array}$ \\
\hline
\end{tabular}

Abbreviation: SAR = specific absorption rate. ${ }^{a}$ The study subjects analysed here were limited to the cases with regular mobile phone use whose maximal SAR distribution was estimated. bypothetical tumour location on the opposite side of the actual tumour across the sagittal plane.

We masked the study's purpose during recruitment, since this could have affected participation differentially among cases and controls, with consequently a biased risk estimate. During the random digit dialling control selection, we tried to ensure that this did not vary between those who were regularly at home and those who were out more often: mobile phone users tend to be more active and busier, staying at home for shorter periods than nonusers, and risk could be overestimated if controls included an unbalanced number of non-users. Although participation among controls in the full interview remained around 50\%, we were able to examine its impact on risk estimates by using the information obtained from the brief questionnaire completed by nonparticipating controls. The age- and sex-adjusted proportions of regular mobile phone users among the control candidates who did not participate in the full interview but agreed to respond to the brief questionnaire $(28.8 \%$ of eligible controls) were $66.4 \%$ (glioma) and $55.1 \%$ (meningioma) as compared with $65.0 \%$ (glioma) and $51.5 \%$ (meningioma) for the controls who participated in the full, face-to-face interview (51.2\% of eligible controls), respectively, indicating that the interviewed controls did not constitute a biased sample in terms of mobile phone use. Adjusted ORs for regular mobile phone users did not vary significantly, irrespective of whether the controls were taken as those who responded to the brief questionnaire or those who took part in the full interview (the ORs were 1.21 for glioma and 0.70 for meningioma).

To date, several case-control studies have been reported from the United States (Muscat et al, 2000; Inskip et al, 2001), Finland (Auvinen et al, 2002), and Sweden (Hardell et al, 1999, 2002, 2003, 2004) before the INTERPHONE study. Two US studies showed negative results for the overall risk of brain tumours, whereas in a Finnish population register-based study, the OR for glioma with relation to ever use of analogue mobile phones was 2.1 (95\% CI: $1.3-3.4)$. Risk was estimated for the left/right laterality of the tumour or for the affected lobe in relation to that of mobile phone use, but no increased risk was observed for tumours on the same side as phone use. In a series of Swedish studies, the risk of tumours in the temporal area on the same side as that used for mobile phone calls was increased for analogue phones, OR 2.3 (95\% CI: 1.2-4.1). However, no increased risks by histological types were observed (Hardell et al, 2003).

Four studies from the INTERPHONE study showed no increased overall risk of glioma or meningioma in relation to regular mobile phone use (Christensen et al, 2004, 2005; Hepworth et al, 2006; Schuz et al, 2006), although glioma risk increased non-significantly $(\mathrm{OR}=2.20 ; 95 \% \mathrm{CI}: 0.94-5.11)$ among long-term users (10 years or more), but no excess risk was found for temporal lobe tumours, considered to be exposed to the highest radio frequency (RF)EMF (Schuz et al, 2006). In a Swedish study, the OR for glioma on the same side as mobile phone use increased to 1.8 (95\% CI: $0.8-$ 3.9) among long-term ( $\geqslant 10$ years) mobile phone users, but the corresponding OR for glioma on the opposite side was found to decrease to 0.6 (95\% CI: $0.3-1.4$ ) among long-term users (Lönn et al, 2005). In a UK study, the OR for glioma on the same side as mobile phone use was 1.24 (95\% CI: $1.02-1.52$ ), but for gliomas on the opposite side, it decreased significantly to 0.75 (95\% CI: $0.61-$ 0.93) (Hepworth et al, 2006). In both studies, it was suggested that such 'complementary risks' above and below unity could reflect recall bias, in which glioma patients tended to recall that they used mobile phones on the same side as the tumour location simply because they knew where the tumour was. In summary, the findings to date on mobile phone use and glioma and meningioma risks are inconsistent.

Most studies of the non-thermal effects of RF-EMF indicate no direct DNA effects such as mutagenicity or genotoxicity of RFEMF exposure in the range of $800-1900 \mathrm{MHz}$ and an SAR of less than $2 \mathrm{~W} \mathrm{~kg}^{-1}$. There is also little evidence for indirect DNA effects, including alterations in gene expression, cell proliferation, or apoptosis (Laszlo et al, 2005; Chauhan et al, 2006; Lixia et al, 2006; Qutob et al, 2006; Joubert et al, 2007). Thus, laboratory studies do not support the possibility that mobile phone use increases the risk of brain tumours.

In conclusion, we observed no increase in overall risk of glioma or meningioma in relation to regular mobile phone use among our Japanese subjects.

\section{ACKNOWLEDGEMENTS}

The study conducted in Japan was fully funded by the Ministry of Internal Affairs and Communications of Japan. We appreciate with grateful cooperation of the surgeons and the staffs of the participating institutions.

\section{Competing interest}

None. 


\section{REFERENCES}

Auvinen A, Hietanen M, Luukkonen R, Koskela RS (2002) Brain tumors and salivary gland cancers among cellular telephone users. Epidemiology 13: $356-359$

Blettner M, Berg G (2000) Are mobile phones harmful? Acta Oncol 39: 927 - 930

Cardis E, Kilkenny M (1999) International case-control study of adult brain, head and neck tumours: results of the feasibility study. Radiat Prot Dosimetry 83: $179-183$

Cardis E, Richardson L, Deltour I, Armstrong B, Feychting M, Johansen C, Kilkenny M, McKinney P, Modan B, Sadetzki S, Schuz J, Swerdlow A, Vrijheid M, Auvinen A, Berg G, Blettner M, Bowman J, Brown J, Chetrit A, Christensen HC, Cook A, Hepworth S, Giles G, Hour M, Iavarone I, Jarus-Hakak A, Klaeboe L, Krewski D, Lagorio S, Lonn S, Mann S, McBride M, Muir K, Nadon L, Parent ME, Pearce N, Salminen T, Schoemaker M, Schlehofer B, Siemiatycki J, Taki M, Takebayashi T, Tynes T, van Tongeren M, Vecchia P, Wiart J, Woodward A, Yamaguchi N (2007) The INTERPHONE study: design, epidemiological methods, and description of the study population. Eur J Epidemiol 22: 647-664

Chauhan V, Mariampillai A, Gajda GB, Thansandote A, McNamee JP (2006) Analysis of proto-oncogene and heat-shock protein gene expression in human derived cell-lines exposed in vitro to an intermittent $1.9 \mathrm{GHz}$ pulse-modulated radiofrequency field. Int J Radiat Biol 82: 347-354

Christensen HC, Schuz J, Kosteljanetz M, Poulsen HS, Boice Jr JD, McLaughlin JK, Johansen C (2005) Cellular telephones and risk for brain tumors: a population-based, incident case-control study. Neurology 64: 1189-1195

Christensen HC, Schuz J, Kosteljanetz M, Poulsen HS, Thomsen J, Johansen C (2004) Cellular telephone use and risk of acoustic neuroma. Am J Epidemiol 159: 277-283

Hardell L, Mild KH, Carlberg M (2002) Case-control study on the use of cellular and cordless phones and the risk for malignant brain tumours. Int J Radiat Biol 78: 931 -936

Hardell L, Mild KH, Carlberg M (2003) Further aspects on cellular and cordless telephones and brain tumours. Int J Oncol 22: 399-407

Hardell L, Mild KH, Carlberg M, Hallquist A (2004) Cellular and cordless telephone use and the association with brain tumors in different age groups. Arch Environ Health 59: $132-137$

Hardell L, Nasman A, Pahlson A, Hallquist A, Hansson Mild K (1999) Use of cellular telephones and the risk for brain tumours: a case-control study. Int J Oncol 15: $113-116$

Hepworth SJ, Schoemaker MJ, Muir KR, Swerdlow AJ, van Tongeren MJ, McKinney PA (2006) Mobile phone use and risk of glioma in adults: case-control study. BMJ 332: $883-887$

Hours M, Bernard M, Montestrucq L, Arslan M, Bergeret A, Deltour I, Cardis E (2007) [Cell phones and risk of brain and acoustic nerve tumours: the French INTERPHONE case-control study]. Rev Epidemiol Sante Publique 55: $321-332$

Inskip PD, Tarone RE, Hatch EE, Wilcosky TC, Shapiro WR, Selker RG, Fine HA, Black PM, Loeffler JS, Linet MS (2001) Cellular-telephone use and brain tumors. $N$ Engl J Med 344: 79-86

International Commission on Non-Ionizing Radiation Protection (1998) Guidelines for limiting exposure to time-varying electric, magnetic, and electromagnetic fields (up to $300 \mathrm{GHz}$ ). Health Phys 74: 494-522

Joubert V, Leveque P, Cueille M, Bourthoumieu S, Yardin C (2007) No apoptosis is induced in rat cortical neurons exposed to GSM phone fields. Bioelectromagnetics 28: 115-121

Laszlo A, Moros EG, Davidson T, Bradbury M, Straube W, Roti Roti J (2005) The heat-shock factor is not activated in mammalian cells exposed to cellular phone frequency microwaves. Radiat Res 164: $163-172$

Lixia S, Yao K, Kaijun W, Deqiang L, Huajun H, Xiangwei G, Baohong W, Wei Z, Jianling L, Wei W (2006) Effects of $1.8 \mathrm{GHz}$ radiofrequency field on DNA damage and expression of heat shock protein 70 in human lens epithelial cells. Mutat Res 602: $135-142$

Lönn S, Ahlbom A, Hall P, Feychting M (2005) Long-term mobile phone use and brain tumor risk. Am J Epidemiol 161: 526-535

Mochizuki SWS, Wake K, Taki M, Yamanaka Y, Shirai H (2002) Detailed dosimetric assessment of a human head exposed to near-field various sources using advanced numerical hybrid techniques. Proceeding of 2002 URSI 27th General Assembly, KA.P.5. The International Union of Radio Science: Maastricht, The Netherlands

Muscat JE, Malkin MG, Thompson S, Shore RE, Stellman SD, McRee D, Neugut AI, Wynder EL (2000) Handheld cellular telephone use and risk of brain cancer. JAMA 284: $3001-3007$

Nagaoka T, Watanabe S, Sakurai K, Kunieda E, Watanabe S, Taki M, Yamanaka Y (2004) Development of realistic high-resolution whole-body voxel models of Japanese adult males and females of average height and weight, and application of models to radio-frequency electromagneticfield dosimetry. Phys Med Biol 49: 1-15

Qutob SS, Chauhan V, Bellier PV, Yauk CL, Douglas GR, Berndt L, Williams A, Gajda GB, Lemay E, Thansandote A, McNamee JP (2006) Microarray gene expression profiling of a human glioblastoma cell line exposed in vitro to a $1.9 \mathrm{GHz}$ pulse-modulated radiofrequency field. Radiat Res 165: 636-644

Repacholi MH (1998) Low-level exposure to radiofrequency electromagnetic fields: health effects and research needs. Bioelectromagnetics 19: $1-19$

Rothman KJ (2000) Epidemiological evidence on health risks of cellular telephones. Lancet 356: $1837-1840$

Rothman KJ, Chou CK, Morgan R, Balzano Q, Guy AW, Funch DP, PrestonMartin S, Mandel J, Steffens R, Carlo G (1996) Assessment of cellular telephone and other radio frequency exposure for epidemiologic research. Epidemiology 7: 291-298

Schuz J, Bohler E, Berg G, Schlehofer B, Hettinger I, Schlaefer K, Wahrendorf J, Kunna-Grass K, Blettner M (2006) Cellular phones, cordless phones, and the risks of glioma and meningioma (Interphone Study Group, Germany). Am J Epidemiol 163: 512-520

Takebayashi T, Akiba S, Kikuchi Y, Taki M, Wake K, Watanabe S, Yamaguchi N (2006) Mobile phone use and acoustic neuroma risk in Japan. Occup Environ Med 63: $802-807$

Varsier N, Wake K, Taki M, Watanabe S, Takebayashi T, Yamaguchi N, Kikuchi Y (2007) SAR characterization inside intracranial tumors for case-control epidemiological studies on cellular phones and RF exposure. Annales des Telecommunications, (in press)

Violanti JM, Marshall JR (1996) Cellular phones and traffic accidents: an epidemiological approach. Accid Anal Prev 28: 265-270

Wake K, Watanabe S, Taki M (2005) Estimation of 3D SAR distributions from mobile phone compliance testing data for the local exposure assessment in epidemiological study. URSI XXVIIIth General Assembly, KA-5. The International Union of Radio Science: New Delhi, India

Wake K, Watanabe S, Taki M (2006) Development of four typical SAR distribution in a human head in the proximity of a cellular phone for an exposure assessment for an epidemiological study. 28th Annual Meeting of the Bioelectromagnetics Society, PA-25 36-37 